\title{
AMP-activated protein kinase is activated in adipose tissue of individuals with type 2 diabetes treated with metformin: a randomised glycaemia-controlled crossover study
}

\author{
J. G. Boyle • P. J. Logan • G. C. Jones • M. Small • \\ N. Sattar • J. M. C. Connell • S. J. Cleland • I. P. Salt
}

Received: 17 January 2011 /Accepted: 24 February 2011/Published online: 1 April 2011

(C) Springer-Verlag 2011

\begin{abstract}
Aims/hypothesis The hypoglycaemic actions of metformin have been proposed to be mediated by hepatic AMP-activated protein kinase (AMPK). As the effects of metformin and the role of AMPK in adipose tissue remain poorly characterised, we examined the effect of metformin on AMPK activity in adipose tissue of individuals with type 2 diabetes in a randomised glycaemia-controlled crossover study.

Methods Twenty men with type 2 diabetes (aged 5070 years) treated with diet, metformin or sulfonylurea alone were recruited from North Glasgow University National Health Service Trusts' diabetes clinics and randomised to either metformin or gliclazide for 10 weeks. Randomisation codes, generated by computer, were put into sealed envelopes and stored by the hospital pharmacist. Medication bottles were numbered, and allocation was done in sequence. The participants and investigators were blinded to group assignment. At the end of each phase of therapy adipose biopsy, AMPK activity (primary endpoint) and levels of lipid metabolism and signalling proteins were assessed. In
\end{abstract}

J. G. Boyle • P. J. Logan · N. Sattar · S. J. Cleland • I. P. Salt $(\bowtie)$ Institute of Cardiovascular and Medical Sciences,

College of Medicine, Veterinary and Life Sciences,

Davidson Building, University of Glasgow,

Glasgow G12 8QQ, UK

e-mail: ian.salt@glasgow.ac.uk

G. C. Jones $\cdot$ M. Small

Department of Diabetes, Gartnavel General Hospital,

Glasgow, UK

J. M. C. Connell

Ninewells Hospital and Medical School, University of Dundee, Dundee, UK parallel, the effect of metformin on AMPK and insulinsignalling pathways was investigated in 3T3-L1 adipocytes. Results Ten participants were initially randomised to metformin and subsequently crossed over to gliclazide, while ten participants were initially randomised to gliclazide and subsequently crossed over to metformin. No participants discontinued the intervention and the adipose tissue AMPK activity was analysed in all 20 participants. There were no adverse events or side effects in the study group. Adipose AMPK activity was increased following metformin compared with gliclazide therapy $(0.057 \pm 0.007$ vs $0.030 \pm 0.005[$ mean $\pm \mathrm{SEM}] \mathrm{nmol} \mathrm{min}{ }^{-1}[\mathrm{mg} \text { lysate }]^{-1} ; p<$ 0.005), independent of AMPK level, glycaemia or plasma adiponectin concentrations. The increase was associated with reduced levels of acetyl-CoA carboxylase (ACC) protein and increased ACC Ser80 phosphorylation. In 3T3-L1 adipocytes, metformin reduced levels of ACC protein and stimulated phosphorylation of AMPK Thr172 and hormone-sensitive lipase Ser565.

Conclusions These results provide the first evidence that metformin activates AMPK and reduces ACC protein levels in human adipose tissue in vivo. Future studies are required to assess the role of adipose AMPK activation in the pharmacological effects of metformin.

Trial registration ISRCTN51336867

Funding This work was supported by grants from the British Heart Foundation, TENOVUS-Scotland, the Biotechnology and Biological Sciences Research Council and Diabetes UK.

Keywords Adipose - AMP-activated protein kinase . Biguanide - Diabetes · Glucose transport - Insulin . Metformin 


\begin{tabular}{|c|c|}
\hline \multicolumn{2}{|c|}{ Abbreviations } \\
\hline $\mathrm{ACC}$ & Acetyl-CoA carboxylase \\
\hline AICAR & $\begin{array}{l}\text { 5-Aminoimidazole-4-carboxamide } \\
\text { ribonucleoside }\end{array}$ \\
\hline Akt & $\begin{array}{l}\text { v-Akt murine thymoma viral oncogene } \\
\text { homologue }\end{array}$ \\
\hline AMPK & AMP-activated protein kinase \\
\hline ERK & Extracellular signal-regulated kinase \\
\hline FAS & Fatty acid synthase \\
\hline GAPDH & Glyceraldehyde-3-phosphate dehydrogenase \\
\hline HSL & Hormone-sensitive lipase \\
\hline $\operatorname{PPAR} \gamma$ & Peroxisome proliferator-activated receptor $\gamma$ \\
\hline
\end{tabular}

\section{Introduction}

Despite the use of metformin as a hypoglycaemic therapy for type 2 diabetes in the UK for more than 50 years, only recently has a potential molecular mechanism of action been proposed, involving AMP-activated protein kinase (AMPK) [1, 2]. The principal hypoglycaemic action of metformin is thought to be the suppression of hepatic gluconeogenesis, yet the precise site(s) and mechanisms of metformin action remain uncertain. Indeed, it has been reported that the effects of metformin go beyond improving $\mathrm{HbA}_{1 \mathrm{c}}$ and may include reductions in cardiovascular endpoints in type 2 diabetes [3].

AMPK is a serine/threonine protein kinase with a central role in the regulation of energy balance at both the cellular and whole body levels (reviewed in Hardie, and in Zhang et al.) $[4,5]$. Stimulation of AMPK in rodents inhibits hepatic glucose production and stimulates insulin-independent glucose uptake in muscle, thereby mimicking insulin [4, 5]. In addition, AMPK activation serves to: (1) inhibit hepatic fatty acid and cholesterol synthesis; (2) stimulate fatty acid oxidation in the liver and muscle; and (3) stimulate mitochondrial biogenesis in muscle $[4,5]$. AMPK is activated in vivo by exercise in muscle and also mediates, at least in part, the insulin-sensitising actions of the adipocytokine, adiponectin [5]. Stimulation of AMPK in patients with type 2 diabetes may, therefore, reduce the associated and detrimental increased lipid biosynthesis and mitochondrial dysfunction [5].

The role of AMPK stimulation in liver and muscle is well characterised, yet the role of AMPK in the other principal insulin-sensitive tissue, adipose, remains poorly defined. AMPK phosphorylates and inhibits acetyl-CoA carboxylase (ACC), reducing fatty acid synthesis in adipocytes [6]. Furthermore, AMPK phosphorylation of rodent hormone-sensitive lipase (HSL) at Ser565 (Ser554 in the human sequence) prevents activation at Ser563 by lipolytic stimuli, such that AMPK activation has been proposed to mimic the anti-lipolytic action of insulin [6-8]. The precise function of AMPK in lipolysis is, however, unclear $[9,10]$, as adrenergic stimulation has been reported to activate AMPK in adipose tissue after exercise [10] and isoprenaline-stimulated AMPK activity has been proposed to be downstream of the stimulation of lipolysis itself [11]. Adipocytes have been reported to be smaller in mice lacking the AMPK $\alpha 1$ catalytic subunit isoform, supporting an anti-lipolytic action of AMPK [9], yet mice lacking the AMPK $\alpha 2$ isoform have been reported to be obese through enlargement of pre-existing adipocytes [12]. Prolonged AMPK activation has been reported to inhibit differentiation of pre-adipocytes into mature adipocytes with an associated reduced protein production of several enzymes concerned with lipid metabolism, namely ACC, fatty acid synthase (FAS) and peroxisome proliferator-activated receptor (PPAR) $\gamma$ [13]. In addition, we and others have reported that AMPK activation is associated with reduced insulinstimulated glucose transport in adipocytes [14-16], although a conflicting study has reported no effect of AMPK stimulation when assessing translocation of the insulinsensitive glucose transporter, GLUT4 [17]. Several physiological and pharmacological stimuli have been reported to stimulate rodent adipose tissue AMPK, including exercise, starvation, troglitazone and adrenaline (epinephrine) [10, 1820], whereas AMPK is inhibited in response to ghrelin, corticosterone and a high-fat diet [21-23] in vivo. However, only one study has investigated AMPK activity in human adipose in vivo; in this study, insulin-resistant patients with Cushing's syndrome exhibited reduced AMPK activity compared with controls [24].

Several studies in cultured cells and animals have reported that AMPK activation mediates the suppression of gluconeogenesis by metformin [1, 2], yet recent contrasting evidence has indicated an AMPK-independent mechanism of action $[25,26]$. There is a dearth of published studies concerning the effect of metformin on AMPK in human tissues in vivo. One study has reported that metformin increased AMPK activity in muscle of individuals with type 2 diabetes [27], potentially leading to improvements in both insulin sensitivity and glycaemia, yet the effect of metformin on AMPK activity in human adipose tissue in vivo has not been investigated. In the current study, the effect of metformin on human adipose tissue AMPK was examined in individuals with type 2 diabetes using a randomised double-blind glycaemiacontrolled crossover design. In addition, the effect of metformin on AMPK activity in cultured 3T3-L1 adipocytes was assessed. 


\section{Methods}

Materials Sheep anti-AMPK $\alpha$ and anti-ACC antibodies were a generous gift from D. G. Hardie (University of Dundee, Dundee, UK). Metformin was obtained from Sigma-Aldrich (Gillingham, Dorset, UK). 2-Deoxy-D- $\left[{ }^{3} \mathrm{H}\right]$ glucose was obtained from Perkin Elmer (Beaconsfield, Buckinghamshire, UK). Mouse anti-glyceraldehyde-3phosphate dehydrogenase (GAPDH) antibodies were from Ambion (Huntingdon, UK). Mouse anti-FAS antibodies were from BD Transduction Laboratories (Oxford, UK). Rabbit anti-HSL, anti-phospho-HSL-Ser565 (human sequence Ser554), anti-extracellular signal-regulated kinase (ERK)1/2; anti-phospho-ERK1/2, anti-IRS1 and anti-IRS2 antibodies were from New England Biolabs (UK) (Hitchin, UK). Mouse anti-PPAR $\gamma$ antibodies were from Santa Cruz Biotechnology (Santa Cruz, CA, USA). Rabbit anti-GLUT4 antibodies were from Synaptic Systems (Goettingen, Germany). All other reagents were from sources described previously $[14,28]$.

Clinical study Twenty men aged 50-70 years with type 2 diabetes (duration $>6$ months) were recruited from diabetes clinics of the North Glasgow University National Health Service Trusts. The North Glasgow University Hospitals National Health Service Trust Ethics Committee approved the study, and all individuals gave written informed consent.

Inclusion criteria included $\mathrm{HbA}_{1 \mathrm{c}}>7 \%$ and $<11 \%$ (DCCT aligned) at screening, BMI range $27-40 \mathrm{~kg} / \mathrm{m}^{2}$ and treatment with diet alone or oral monotherapy (metformin or sulfonylurea). Exclusion criteria included warfarin treatment, patients treated with insulin currently or in the previous 12 months, previous intolerance of metformin or sulfonylurea, presence of a contraindication to metformin therapy, poorly controlled hypertension (systolic BP > $170 \mathrm{mmHg}$, diastolic $\mathrm{BP}>100 \mathrm{mmHg}$ ) and a cardiovascular event (i.e. ECG/troponin proven myocardial infarction or cerebrovascular accident) in the previous 6 months. The study had a randomised glycaemia-controlled crossover design whereby individuals discontinued medication during a 6week run-in period and were subsequently randomised to metformin (500 $\mathrm{mg}$ three times daily) or gliclazide (80 mg twice daily with a lunchtime matching placebo capsule to ensure blinding) for 10 weeks. Following a 6week washout phase, the groups were crossed over. Study participants and investigators were blinded to group assignment. Randomisation and tablet supply were coordinated by the hospital pharmacy, which provided metformin and gliclazide as capsules of identical appearance.

Individuals attended for a brief assessment at 5 weeks during each phase to identify any side effects and assess glycaemia control. Individuals subsequently attended at the end of each 10-week study phase (in the fasting condition having abstained from alcohol, caffeine and moderate/ heavy exercise in the preceding $24 \mathrm{~h}$ ) for clinical measurements, blood sampling for biochemical analysis and an adipose tissue biopsy (Fig. 1).

Clinical measures Body weight was measured using analogue scales to within $500 \mathrm{~g}$ in light clothing; height was measured barefoot using a stadiometer to within $0.5 \mathrm{~cm}$. BP recordings were taken by a semi-automatic sphygmomanometer (Dinamap, GE Healthcare, Bracknell, UK) in the recumbant position for $30 \mathrm{~min}$. The mean of three measurements was calculated. Measurements of plasma glucose, insulin, $\mathrm{HbA}_{1 \mathrm{c}}$ and lipid concentrations were performed in the routine clinical laboratory using an automated analyser (Abbott Diagnostics, Maidenhead, UK). Total adiponectin was determined by ELISA (R\&D Systems, Abingdon, UK) as described previously by Nelson et al. [29].

Adipose tissue biopsy and lysate preparation A 2 g portion of adipose tissue was cut from the margin of a gluteal biopsy, snap frozen in liquid nitrogen and stored at $-80^{\circ} \mathrm{C}$. When all samples had been collected the samples were thawed for batch analysis. Adipose lysates were prepared by homogenisation ( 20 passes) in 1 volume of immunoprecipitation buffer $\left(50 \mathrm{mmol} / \mathrm{l}\right.$ Tris- $\mathrm{HCl}, \mathrm{pH} 7.4$ at $4{ }^{\circ} \mathrm{C}$; $150 \mathrm{mmol} / 1 \mathrm{NaCl} ; 50 \mathrm{mmol} / \mathrm{l} \mathrm{NaF} ; 5 \mathrm{mmol} / 1 \mathrm{Na}_{4} \mathrm{P}_{2} \mathrm{O}_{7}$; $1 \mathrm{mmol} / 1$ sodium vanadate; $1 \mathrm{mmol} / \mathrm{l}$ EDTA; $1 \mathrm{mmol} / \mathrm{l}$ EGTA; 1\% [vol./vol.] glycerol; 1\% [vol./vol.] Triton X-100; $1 \mathrm{mmol} / \mathrm{l}$ dithiothreitol; $0.1 \mathrm{mmol} / \mathrm{l}$ benzamidine; $0.1 \mathrm{mmol} / 1$ phenylmethylsulfonyl fluoride; $5 \mathrm{mg} / \mathrm{l}$ soyabean trypsin inhibitor) in a Dounce homogeniser. Lysates were centrifuged $\left(22,000 \mathrm{~g}, 4^{\circ} \mathrm{C}, 5 \mathrm{~min}\right)$ and the resulting infranatant fraction was aspirated and stored at $-80^{\circ} \mathrm{C}$ prior to use.

3T3-L1 cell culture and lysate preparation 3T3-L1 fibroblasts were cultured and differentiated, and lysates were prepared from adipocytes (8-12 days post-differentiation) as described previously by Salt et al. [14].

SDS-PAGE and western blotting Adipose lysates or 3T3L1 adipocyte lysates were resolved by SDS-PAGE, transferred to nitrocellulose and subjected to immunoblotting as described previously by Salt et al. [14]. Band intensity was determined by densitometry. ACC, AMPK, HSL, Akt and ERK1/2 phosphorylation are expressed as the ratio of phosphorylated to total protein density.

2-Deoxyglucose transport assays Basal and insulinstimulated glucose uptake in 3T3-L1 fibroblasts or adipo- 
Fig. 1 Flow diagram of clinical study

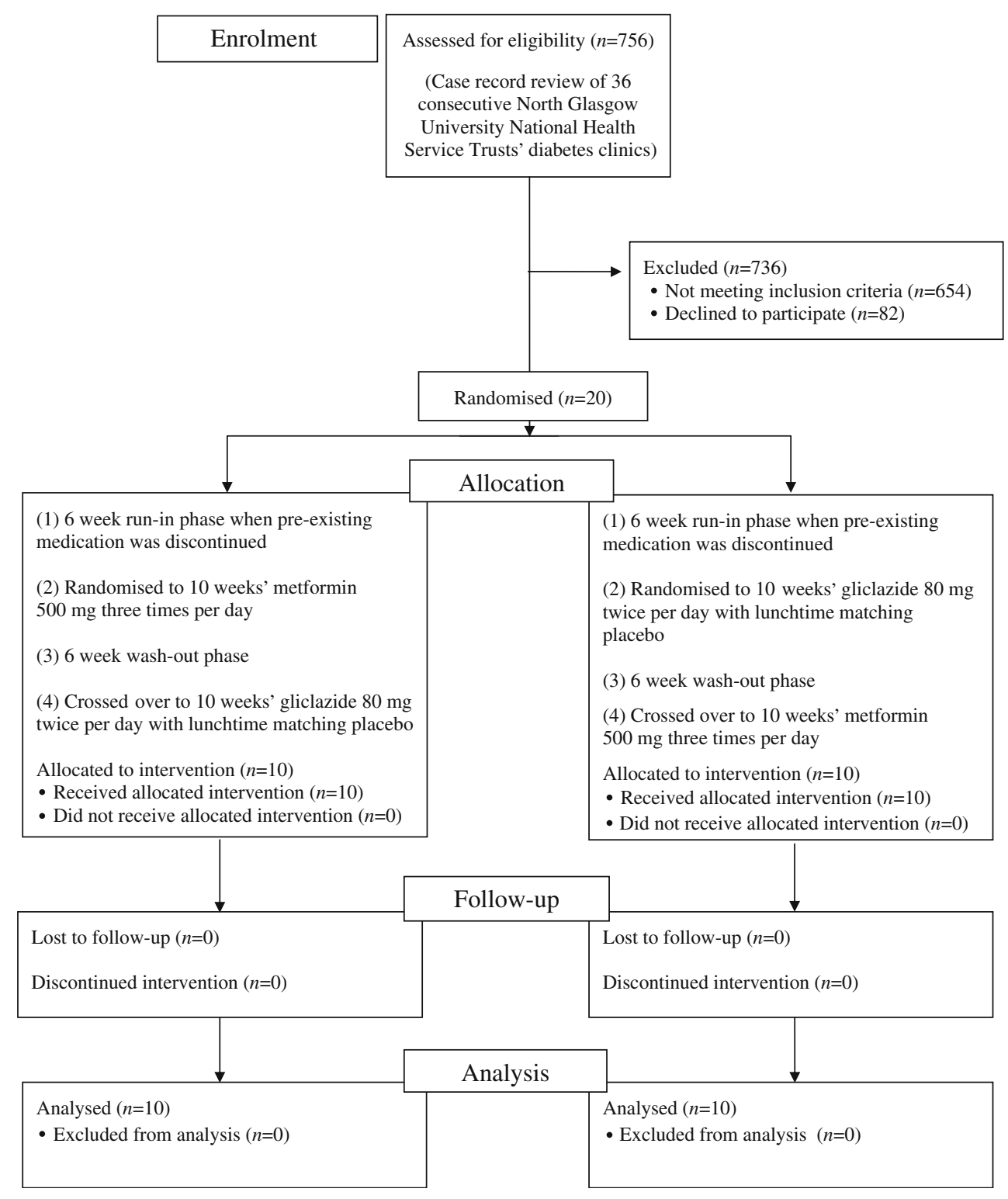

cytes was measured by the uptake of 2-deoxy-D- $\left[{ }^{3} \mathrm{H}\right]$ glucose as described previously by Salt et al. [14]. Nonspecific cell-associated radioactivity was determined in parallel incubations performed in the presence of $10 \mu \mathrm{mol} / 1$ cytochalasin B.

AMPK assays AMPK was immunoprecipitated from human adipose lysates $(100 \mu \mathrm{g})$ and assayed using the SAMS substrate peptide as described previously by Boyle et al. [28]. Experiments were performed in four batches of ten lysates with AMPK activity determined relative to an internal standard (purified rat liver AMPK of known activity) and results are expressed as $n m o l{ }^{32} \mathrm{P}$ incorporated into SAMS peptide $\min ^{-1}$ (mg lysate $)^{-1}$.
Statistical analysis For studies using 3T3-L1 adipocytes, results are expressed as the mean \pm SEM and statistically significant differences were determined using a Student's $t$ test or one-way ANOVA where appropriate, with $p<0.05$ as significant. For clinical data, baseline characteristics are reported as mean and SD. Outcome variables are summarised by treatment as mean (SD). Treatment effect estimates are reported as the mean difference between values recorded after gliclazide and metformin, adjusting for patient and period effects within a fixed-effects linear regression model. Normal probability plots of model residuals were generated to check for serious departures from normality of model residuals. Equivalent values from each study phase were compared using paired Student's 
$t$ tests unless stated otherwise. $p<0.05$ was taken as signifying conventional levels of statistical significance.

\section{Results}

At the time of recruitment, 12 individuals were taking metformin monotherapy, six were diet controlled and two were using gliclazide monotherapy. No individuals dropped out of the study and there were no reports of changes to drug therapy during the study period. The clinical characteristics of the 20 individuals at the time of recruitment and before the first 6 -week run-in period when medication was discontinued are summarised in Table 1.

The effect of each 10-week treatment phase on the clinical characteristics is shown in Table 2. There was no significant difference in BMI, blood pressure, plasma insulin, total cholesterol, HDL-cholesterol or triacylglycerol between the two stages of treatment (Table 2). Gliclazide therapy was more effective at lowering $\mathrm{HbA}_{1 \mathrm{c}}$ than metformin, and was associated with significantly lower fasting blood glucose and LDL-cholesterol levels (Table 2). Plasma adiponectin concentrations were not different between phases of therapy (Table 2). There was a significant approximate twofold increase in AMPK activity in adipose tissue biopsies from individuals after metformin therapy compared with gliclazide therapy (Fig. 2) that was associated with a significant increase in the phosphorylation of AMPK $\alpha$ at Thr172 and the AMPK substrate, ACC at Ser80 (mouse sequence Ser79) (Fig. 3). Although there was a tendency towards increased phosphorylation of HSL at the AMPK site, Ser554 in adipose tissue from individuals treated with metformin, this did not reach significance $(p=$ 0.09).

Total levels of AMPK $\alpha$ or HSL protein (normalised to GAPDH) were unaltered between treatment phases yet ACC protein levels were significantly reduced in adipose tissue lysates from individuals treated with metformin

Table 1 Clinical characteristics at recruitment

\begin{tabular}{ll}
\hline Variable & Mean (SD) \\
\hline Age (years) & $56.5(3.9)$ \\
$\mathrm{HbA}_{1 \mathrm{c}}(\%)$ & $8.31(1.24)$ \\
$\mathrm{BMI}\left(\mathrm{kg} / \mathrm{m}^{2}\right)$ & $31.4(4.3)$ \\
Duration of diabetes (years) & $3.5(2.6)$ \\
\hline
\end{tabular}

Characteristics were determined prior to the first 6-week run-in period when medication was discontinued
(Fig. 3). The level of GAPDH protein was unaltered between treatment phases (metformin $0.947 \pm 0.075$; gliclazide $0.947 \pm 0.073$; arbitrary units mean \pm SEM, $n=19$ ). We also determined the levels of FAS, GLUT4 and PPAR $\gamma$ protein, downregulation of which, like ACC, has been reported to be associated with prolonged AMPK activation in adipocytes $[13,30]$. The levels of FAS, GLUT4 and PPAR $\gamma$ protein were unaltered between treatment phases (Fig. 3).

We next investigated whether the observed alteration in AMPK activity was associated with any changes in insulin and mitogenic signalling pathways. Although there was a tendency towards increased phosphorylation of $\mathrm{v}$-akt murine thymoma viral oncogene homologue (Akt, also known as PKB) at Ser473 in adipose tissue from individuals treated with metformin, this did not reach significance $(p=0.12)$. Furthermore, neither ERK1/2 phosphorylation nor the GAPDH-normalised level of IRS1, IRS2, Akt or ERK $1 / 2$ protein was different between phases of therapy (Fig. 3).

As adipose tissue contains stromal/vascular cells in addition to adipocytes, we next used the 3T3-L1 adipocyte cell line to determine the effects of metformin on adipocyte AMPK. Stimulation of 3T3-L1 adipocytes for $48 \mathrm{~h}$ with metformin caused a significant increase in Thr172 phosphorylation, as did stimulation for $24 \mathrm{~h}$ with the AMPK activator, 5-aminoimidazole-4-carboxamide ribonucleoside (AICAR) (Fig. 4), whereas gliclazide had no effect (data not shown). To determine the effects of metformin on AMPK substrates, we examined ACC and HSL phosphorylation at their AMPK-specific sites. Metformin had no significant effect on ACC Ser79 phosphorylation, yet stimulated HSL phosphorylation after incubation for 24 $48 \mathrm{~h}$ (Fig. 4). In contrast, AICAR markedly and rapidly stimulated ACC and HSL phosphorylation (Fig. 4). Incubation of 3T3-L1 adipocytes with metformin $(48 \mathrm{~h})$ or AICAR $(24 \mathrm{~h})$ led to a significant reduction in total ACC level, yet incubation with either metformin or AICAR had no significant effect on the levels of FAS, GLUT4 or PPAR $\gamma$ (Fig. 4). In addition, neither metformin nor AICAR incubation had any significant effect on the total amount of AMPK $\alpha$ or HSL (data not shown).

Stimulation of 3T3-L1 adipocytes with metformin (24/48 h) or AICAR significantly reduced the fold stimulation in glucose transport elicited by insulin, whereas stimulation with metformin for $30 \mathrm{~min}$ had no effect (Fig. 5a). The reduction in the extent of insulin stimulation elicited by metformin (24 and $48 \mathrm{~h}$ ) was likely attributable to a tendency towards increased basal glucose transport that did not reach significance $(p=$ 0.09 and $p=0.10$, respectively), as insulin-stimulated glucose transport was quantitatively unaltered (Fig. 5b). Incubation of 
Table 2 Effect of treatment on clinical characteristics

\begin{tabular}{|c|c|c|c|c|}
\hline \multirow[t]{2}{*}{ Outcome } & \multicolumn{2}{|c|}{ Raw outcomes by treatment } & \multicolumn{2}{|l|}{ Treatment effect } \\
\hline & Metformin & Gliclazide & Estimate $(95 \% \mathrm{CI})$ & $p$ value \\
\hline BMI $\left(\mathrm{kg} / \mathrm{m}^{2}\right)$ & $31.0 \pm 4.3$ & $31.0 \pm 4.5$ & $-0.1(-0.5,0.4)$ & 0.801 \\
\hline Systolic BP (mmHg) & $137.2 \pm 19.7$ & $137.0 \pm 21.0$ & $-0.3(-4.6,4.1)$ & 0.906 \\
\hline Diastolic BP (mmHg) & $72.3 \pm 9.5$ & $75.0 \pm 11.8$ & $2.8(-1.5,7.0)$ & 0.193 \\
\hline $\mathrm{HbA}_{1 \mathrm{c}}(\%)$ & $8.34 \pm 1.70$ & $7.82 \pm 1.74$ & $-0.53(-0.77,-0.28)$ & $<0.001$ \\
\hline Glucose $(\mathrm{mmol} / \mathrm{l})$ & $12.1 \pm 4.7$ & $10.3 \pm 3.2$ & $-1.8(-3.0,-0.6)$ & 0.005 \\
\hline Insulin (pmol/l) & $93.6 \pm 82.8$ & $85.8 \pm 36.0$ & $-7.8(-48.0,32.4)$ & 0.69 \\
\hline Cholesterol (mmol/l) & $5.16 \pm 1.16$ & $4.75 \pm 1.59$ & $-0.40(-0.81,0.01)$ & 0.054 \\
\hline HDL-cholesterol (mmol/l) & $1.25 \pm 0.24$ & $1.20 \pm 0.24$ & $-0.05(-0.12,0.02)$ & 0.131 \\
\hline LDL-cholesterol (mmol/l) & $2.79 \pm 0.74$ & $2.42 \pm 0.73$ & $-0.40(-0.73,-0.06)$ & 0.022 \\
\hline Triacylglycerols (mmol/l) & $2.78 \pm 3.76$ & $2.94 \pm 4.80$ & $0.16(-0.42,0.73)$ & 0.576 \\
\hline Adiponectin $(\mu \mathrm{g} / \mathrm{ml})$ & $4.19 \pm 2.67$ & $4.65 \pm 3.32$ & $0.46(-0.01,0.92)$ & 0.054 \\
\hline
\end{tabular}

3T3-L1 adipocytes with metformin (up to $48 \mathrm{~h}$ ) had no effect on cell viability, as assessed by 3-(4,5-dimethylthiazol-2-yl)2,5-diphenyltetrazolium bromide (MTT) assay (data not shown). In 3T3-L1 adipocytes stimulated with metformin for $0.5 \mathrm{~h}$, there was a modest yet significant quantitative increase in insulin-stimulated glucose transport, and AICAR elicited a marked significant quantitative decrease in insulinstimulated glucose transport (Fig. 5b).

To assess whether metformin altered glucose transport in fibroblasts, we examined 2-deoxyglucose uptake in undifferentiated 3T3-L1 fibroblasts after incubation with either insulin (15 min, $1 \mu \mathrm{mol} / \mathrm{l})$ and/or metformin $(0.5-48 \mathrm{~h}$, $1 \mathrm{mmol} / \mathrm{l})$. Neither metformin nor insulin had any effect on 2-deoxyglucose transport in 3T3-L1 fibroblasts (data not shown). We next examined the effect of metformin on basal and insulin-stimulated phosphorylation of Akt. Prolonged stimulation with metformin had no significant effect on the
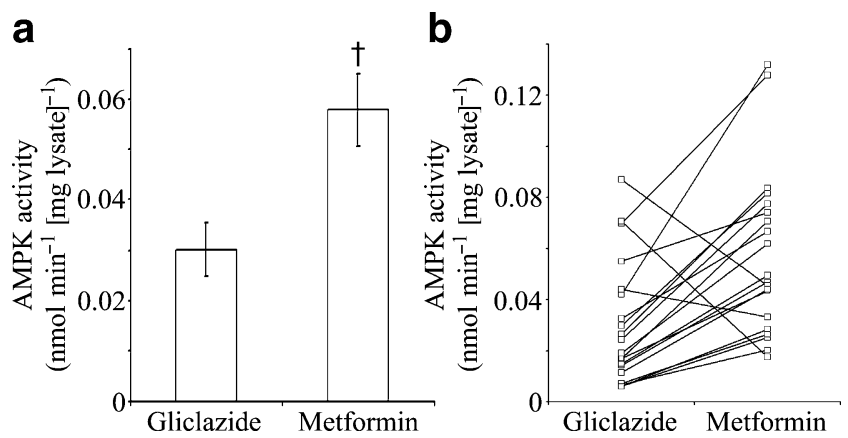

Fig. 2 The effect of metformin monotherapy on adipose tissue AMPK activity. Lysates were prepared from adipose biopsies at the end of each study phase. Total AMPK (using anti-AMPK $\alpha 1$ and antiAMPK $\alpha 2$ antibodies) was immunoprecipitated from lysates and assayed for AMPK activity in batches relative to an internal control (purified rat liver AMPK) of known activity. Data shown represent: (a) the mean \pm SEM AMPK activity and (b) AMPK activity in each individual. ${ }^{\dagger} p<0.005$ relative to gliclazide level of Akt protein or either the basal or insulin-stimulated phosphorylation of Akt at Ser473 (Fig. 5c,d).

\section{Discussion}

The key finding of this study is that, when compared with gliclazide, metformin activates AMPK in human adipose tissue in vivo. To our knowledge, this represents only the second study to demonstrate activation of AMPK by metformin in human tissues in vivo and the first to demonstrate this relationship in adipose tissue. The increase in adipose AMPK activity was associated with an increase in AMPK Thr172 phosphorylation, whereas the total level of AMPK protein was unaltered, indicating an increase in the specific activity of AMPK. Adiponectin has been demonstrated to stimulate AMPK in multiple tissues, including isolated rat adipocytes [31], yet the increased AMPK activity in individuals treated with metformin was not associated with any significant change in plasma adiponectin concentrations, in agreement with a previous study [32], indicating that the stimulation of AMPK is independent of adiponectin.

As several forms of adiponectin exist, however, we cannot discount the possibility that the increase in AMPK activity is the result of metformin altering the plasma concentrations of a particular form rather than the total adiponectin measured in the current study. Gliclazide had no effect on AMPK activity in 3T3-L1 adipocytes and has been reported to increase plasma adiponectin concentrations in individuals with type 2 diabetes [33], whereas we have shown that metformin is capable of directly stimulating 3T3-L1 adipocyte AMPK activity. This latter observation is in agreement with previous reports in 3T3-L1 adipocytes [34] and isolated human subcutaneous adipocytes stimulated with metformin in vitro [35]. These data argue against the possibility that gliclazide 
Fig. 3 The effect of metformin monotherapy on adipose tissue protein levels. Lysates were prepared from adipose biopsies of individuals treated with gliclazide (white bars) or metformin (black bars) at the end of each study phase.

Adipose biopsy lysates $(50 \mu \mathrm{g})$ were resolved by SDS-PAGE and subjected to immunoblotting with the antibodies indicated. a Representative immunoblots from individuals after gliclazide $(\mathrm{G})$ or metformin (M) therapy, with the positions of markers of known molecular mass shown (kDa). b Densitometric analysis of phosphorylation of AMPK, HSL, ACC, ERK1/2 and Akt was quantified relative to total production in each lysate (mean $\pm \mathrm{SEM}$, $n=12$ ). c Band intensity was quantified relative to GAPDH in each lysate. The amounts of the following are shown (mean \pm SEM): AMPK $\alpha$, IRS1, Akt $(n=19)$; FAS $(n=13)$; and ACC, HSL, ERK1/2, GLUT4, PPAR $\gamma, \operatorname{IRS} 2(n=12) * p<0.05$ relative to gliclazide; $* * p<0.01$ relative to gliclazide a

Anti-p-ACC-S80

Anti-ACC

Anti-p-HSL-S554

Anti-HSL

Anti-p-AMPK-T172

Anti-AMPK $\alpha$

Anti-p-ERK1/2

Anti-ERK1/2

Anti-FAS

Anti-GLUT4

Anti-PPAR $\gamma$

Anti-IRS-1

Anti-IRS-2

Anti-p-Akt-S473

Anti-AKT

Anti-GAPDH

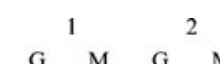

G M G M
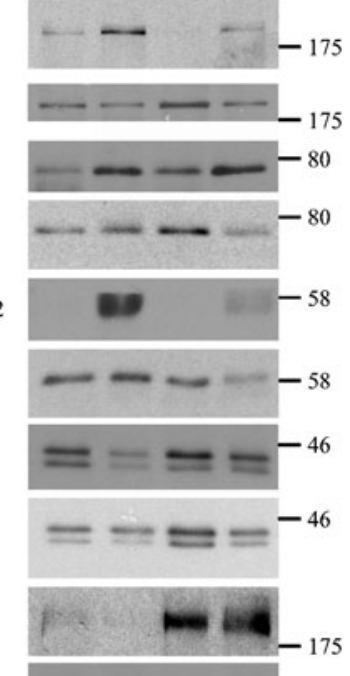

c

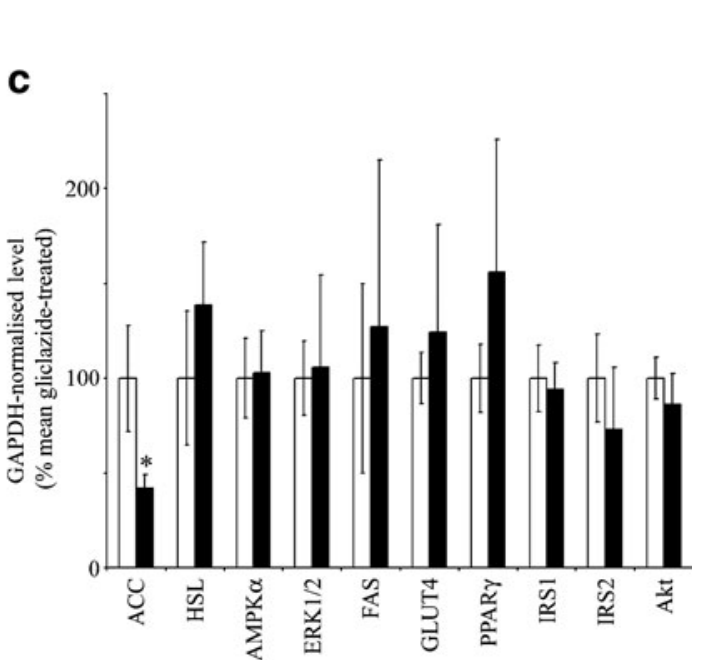

b

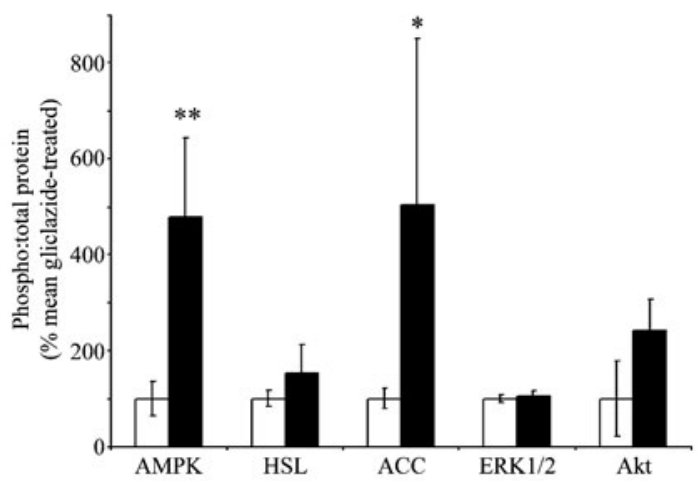

attenuated AMPK activity in the individuals of this study, rather than metformin activating AMPK, and further dissociate the observed increase in adipose AMPK activity from circulating adiponectin concentrations.

Glycaemic control at the time of recruitment and at the end of each treatment phase was suboptimal. We believe that this reflects the fact that many patients with type 2 diabetes require more than one drug to reach their individualised glycaemic targets. The study design aimed to match glycaemic control at the end of each phase to exclude this as a confounding factor. This proved not to be possible, presumably because of imperfect matching of the chosen randomisation doses of metformin and gliclazide. Metformin increased AMPK activity when compared with gliclazide despite improved glycaemia changes with the latter, further supporting a glucose-independent mechanism for the effect of metformin. Associated with the reduced $\mathrm{HbA}_{1 \mathrm{c}}$ after gliclazide therapy was a small improvement in plasma LDL-cholesterol.

In humans, few studies have addressed the molecular consequences of metformin therapy in human adipose tissue in vivo. The increased AMPK activity observed in individuals undertaking metformin treatment was associated with increased
ACC phosphorylation. Phosphorylation at this site inhibits malonyl CoA synthesis, such that fatty acid synthesis is likely to be attenuated. Intriguingly this finding was not recapitulated in 3T3-L1 adipocytes stimulated for up to $48 \mathrm{~h}$ with metformin, yet this may reflect the very different concentrations of, and lengths of exposure to, metformin in either case (approximately $10 \mu \mathrm{mol} / 1$ [36] for 10 weeks in vivo; $1 \mathrm{mmol} / 1$ for $48 \mathrm{~h}$ in vitro). Prolonged AMPK activation has been reported to inhibit differentiation of fibroblasts into adipocytes with an associated reduced level of ACC, FAS and PPAR $\gamma$ protein [13]. In both adipose tissue from metformin-treated individuals and 3T3-L1 adipocytes stimulated with metformin for $48 \mathrm{~h}$ there was a marked reduction in total ACC, yet levels of FAS and PPAR $\gamma$ were unaffected. The lack of effect of metformin on the level of FAS protein is in agreement with a previous study of FAS mRNA expression in adipose tissue of individuals with impaired glucose tolerance [37], and may reflect an inability of AMPK activation to reduce FAS or PPAR $\gamma$ in mature adipocytes when compared with cells differentiating into adipocytes in culture.

Metformin has been reported to reduce subcutaneous adipocyte size in individuals with type 2 diabetes [38] and 


\section{a}

Metformin

AICAR

$\begin{array}{lllllllllllllll}\text { Time (h) } & 0 & 0 & 0.5 & 0.5 & 24 & 24 & 48 & 48 & 0.5 & 0.5 & 24 & 24\end{array}$

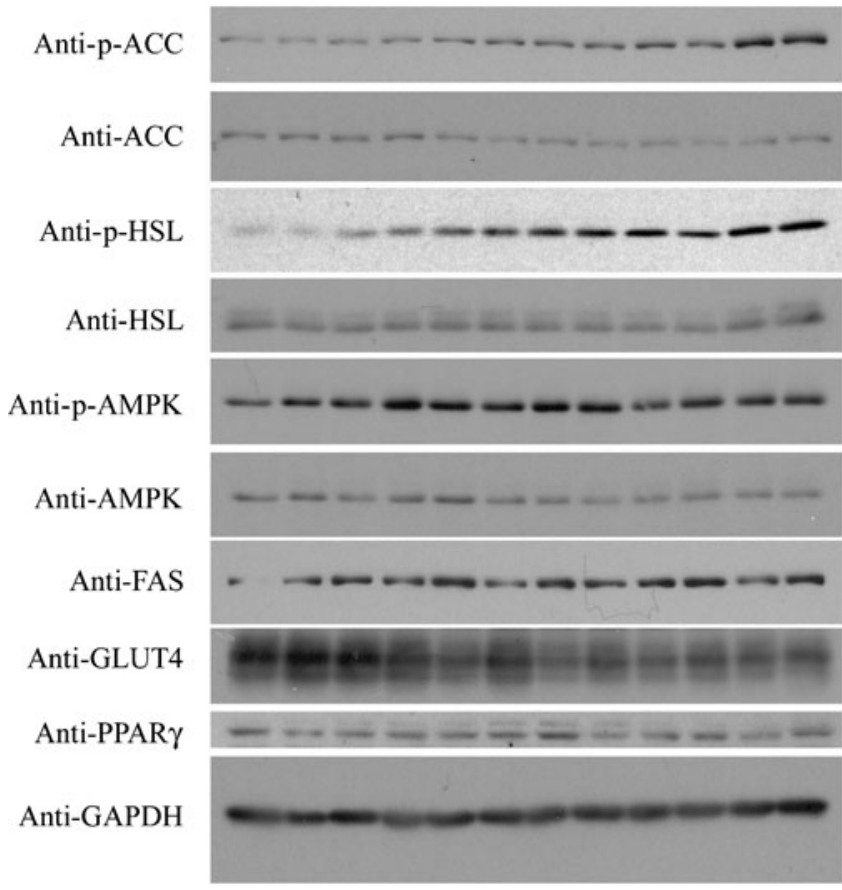

Fig. 4 Effect of metformin on levels of protein and phosphorylation in 3T3-L1 adipocytes. 3T3-L1 adipocytes were incubated in the presence and absence of metformin $(1 \mathrm{mmol} / \mathrm{l})$ or AICAR $(2 \mathrm{mmol} / \mathrm{l})$ for the durations indicated. 3T3-L1 adipocyte lysates $(10 \mu \mathrm{g})$ were resolved by SDS-PAGE and subjected to immunoblotting with the antibodies indicated. a Representative immunoblots are shown, repeated in four additional independent experiments with similar

reduce plasma NEFA concentrations [39], indicating a suppression of lipolysis, yet others have reported no significant effect [40]. No studies have, to the authors' knowledge, compared the effects of sulfonylurea and metformin therapy on lipolysis. The current study is the first to examine the effect of metformin on adipose HSL phosphorylation in vivo. As the modest increase in HSL Ser554 phosphorylation in individuals treated with metformin did not reach significance, it is difficult to draw definite conclusions concerning the role of AMPK-mediated phosphorylation of HSL in any potential suppression of lipolysis. In contrast, incubation of 3T3-L1 adipocytes with metformin caused a robust stimulation of the equivalent phosphorylation site in mouse HSL (Ser565) and a recent study has demonstrated that ex vivo stimulation of isolated human adipocytes with metformin attenuated isoprenaline and atrial natriuretic peptide (ANP)-stimulated lipolysis without affecting basal lipolysis. Furthermore, metformin stimulated HSL Ser554 phosphorylation under ANPstimulated conditions, although the effect of metformin alone was not reported [35]. One explanation for the
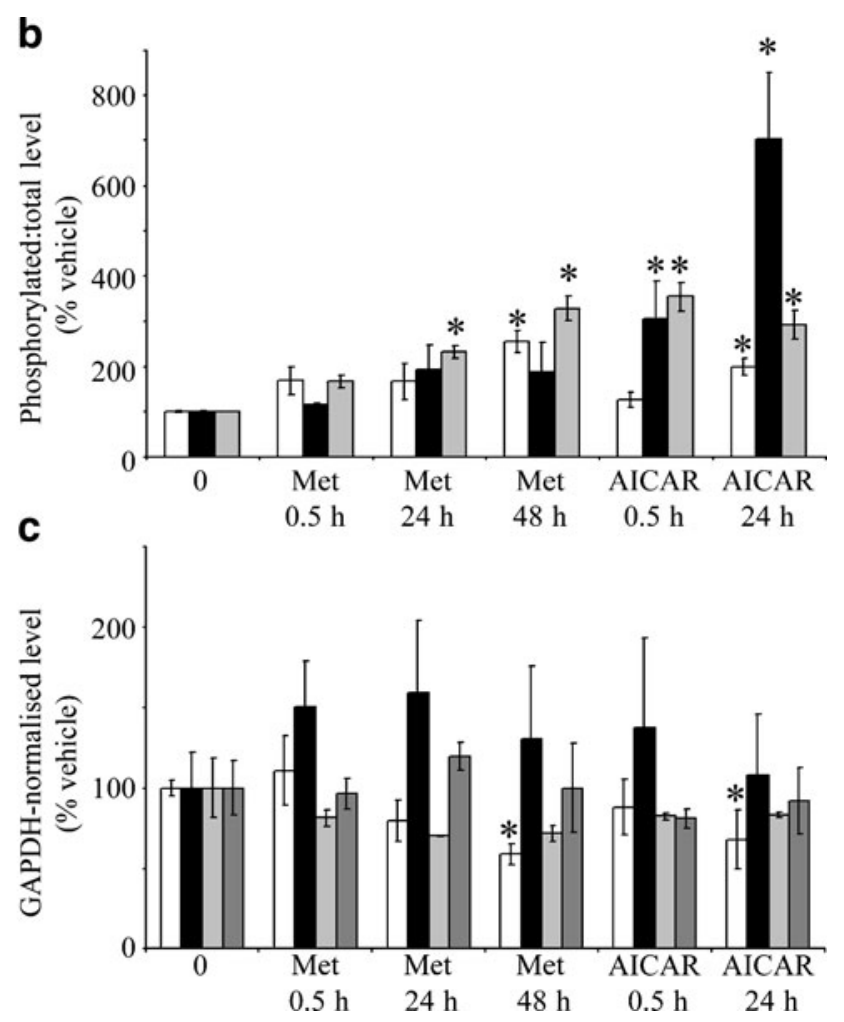

results. b Phosphorylation of AMPK (white bars), HSL (black bars) and ACC (grey bars) was quantified relative to total amount in each lysate (mean \pm SEM, $n=5$ ). c Band intensity of ACC (white bars), FAS (black bars), GLUT4 (light grey bars) and PPAR $\gamma$ (dark grey bars) was quantified relative to GAPDH in each lysate (mean \pm SEM, $n=5$ ). $* p<0.05$ relative to absence of metformin or AICAR. Met, metformin

discrepancy between the effects observed in isolated or cultured cells and our in vivo study is likely to be the differing concentrations of metformin used in each case and the very different durations of exposure.

We have previously reasoned that activation of AMPK is associated with reduced insulin-stimulated glucose transport in adipocytes [14], thereby attenuating fatty acid and triacylglycerol synthesis, conserving ATP for more immediate cellular demands. In agreement with our previous study, AICAR reduced insulin-stimulated glucose transport. In contrast, stimulation with metformin had little effect on quantitative insulin-stimulated glucose transport under conditions where there was concomitant activation of AMPK. Prolonged stimulation with metformin reduced the fold stimulation of glucose transport by insulin, yet this was likely to have occurred because of a non-significant increase in basal glucose transport rather than a decrease in insulin-stimulated glucose transport. A previous study also reported a modest tendency toward increased basal glucose transport in 3T3-L1 adipocytes stimulated with $0.3 \mathrm{mmol} / 1$ metformin for $24 \mathrm{~h} \mathrm{[41].}$ 

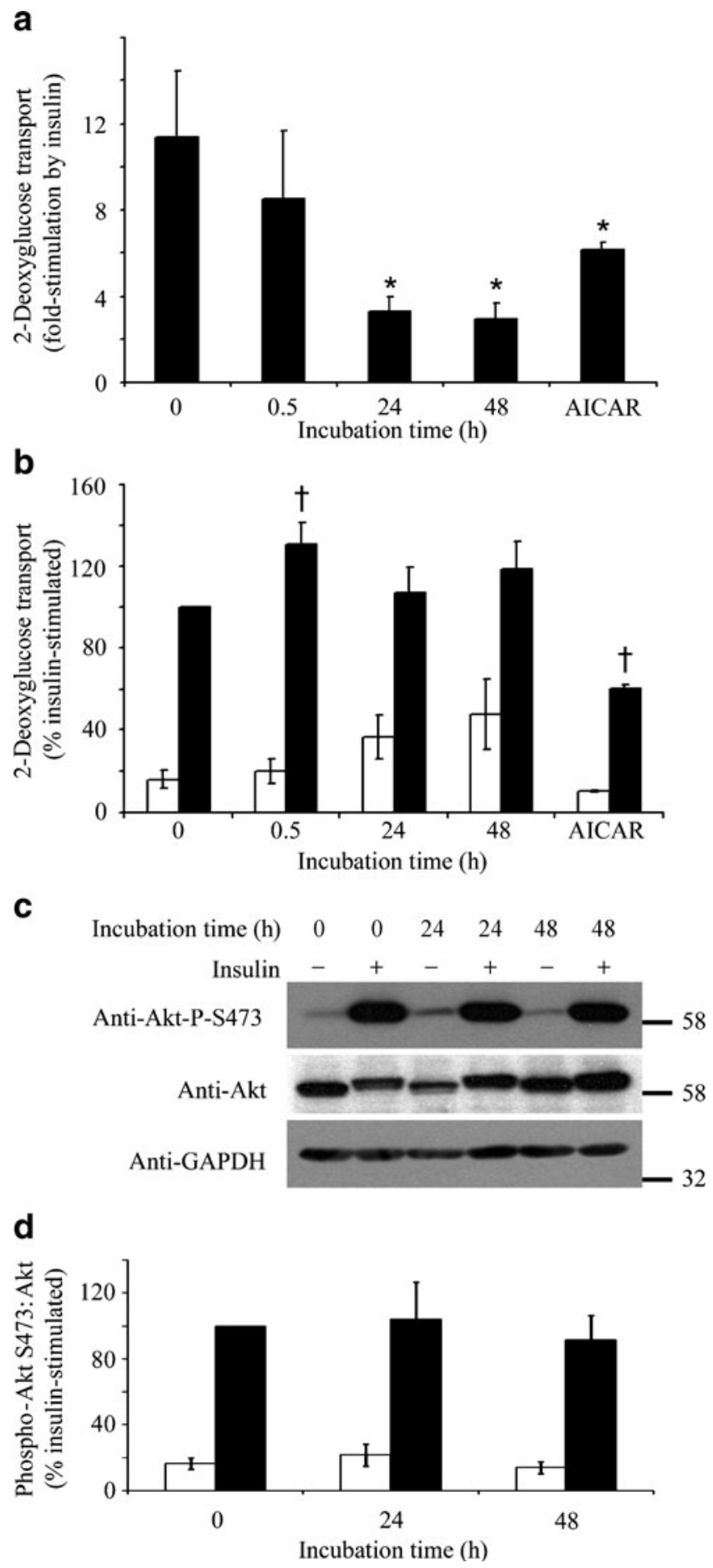

The data in the current study represent, to the authors' knowledge, the first report of the effect of metformin on insulin-stimulated glucose transport in 3T3-L1 adipocytes. Previous studies in human adipocytes differentiated from mesenchymal stem cells or pre-adipocytes indicate that metformin stimulation for $24 \mathrm{~h}$ increased basal and insulinstimulated glucose uptake [22, 42], yet incubation of adipocytes isolated from individuals with type 2 diabetes
Fig. 5 Effect of metformin on glucose transport and insulin signalling in 3T3-L1 adipocytes. a, b 3T3-L1 adipocytes were incubated in the presence and absence of metformin $(1 \mathrm{mmol} / \mathrm{l})$ for the durations indicated or AICAR $(2 \mathrm{mmol} / \mathrm{l})$ for $30 \mathrm{~min}$ prior to incubation in the presence or absence of insulin $(10 \mathrm{nmol} / \mathrm{l})$ for a further $15 \mathrm{~min}$ and 2 deoxyglucose uptake assessed. Data shown represent the mean \pm SEM of (a) fold stimulation by insulin, or (b) basal (white bars) and insulinstimulated (black bars) glucose transport of three independent experiments performed in triplicate. ${ }^{*} p<0.05$ relative to the value in the absence of metformin/AICAR; ${ }^{\dagger} p<0.05$ relative to insulin alone. c, d 3T3-L1 adipocyte lysates were prepared from cells incubated with metformin $(2 \mathrm{mmol} / \mathrm{l})$ for the durations indicated and in the presence (black bars) or absence (white bars) of insulin $(10 \mathrm{nmol} / \mathrm{l})$ for the final $15 \mathrm{~min}$. Lysates were resolved by SDS-PAGE and subjected to immunoblotting with the antibodies indicated. c Representative immunoblots with the positions of markers of known molecular mass shown (kDa). d Basal (white bars) and insulin-stimulated (black bars) phosphorylation of Akt at Ser473 was quantified relative to total amount in each lysate (mean $\pm \operatorname{SEM}, n=3$ )

with metformin for $1 \mathrm{~h}$ has been reported to have no effect on basal or insulin-stimulated glucose uptake [38]. Increased levels of GLUT4 protein have been associated with AMPK activation in cultured adipocytes [30], and metformin has been reported to increase mRNA expression of GLUT4 (also known as $S L C 2 A 4$ ) in adipose from women with polycystic ovary syndrome [43], yet the amount of GLUT4 protein was unaltered in the current study in either human adipose tissue or 3T3-L1 adipocytes, indicating that changes in GLUT4 expression are unlikely to underlie the difference in the fold stimulation by insulin. Furthermore, as metformin did not significantly alter the levels of the insulin signalling pathway proteins IRS1, IRS2 or Akt in human adipose samples or the insulin-stimulated phosphorylation of Akt in 3T3-L1 adipocytes, modulation of insulin signalling is similarly unlikely to underlie the reduction in the fold change in insulinstimulated glucose transport.

Taken together, these data suggest that metformin stimulates adipose AMPK in a glycaemia- and adiponectin-independent fashion in vivo. Metformin stimulates AMPK in 3T3-L1 adipocytes and the metforminstimulated increase in AMPK activity is associated with downregulated ACC activity in both cases. Furthermore, there is a significant increase in HSL phosphorylation (at an AMPK-specific site) in 3T3-L1 adipocytes and a tendency towards phosphorylation at that site in human adipose, whereas metformin is without effect on the levels of FAS, PPAR $\gamma$ or GLUT4 protein in either adipose biopsies from individuals with type 2 diabetes or 3T3-L1 adipocytes. We cannot dismiss the possibility that the observed increase in AMPK activity in human adipose was occurring in the stromal/vascular fraction rather than in adipocytes themselves, as metformin has been reported to activate AMPK in human vascular endothelial cells [44] and a macrophage cell line [45]. As metformin also stimulated AMPK 
phosphorylation whilst reducing total ACC protein in both human adipose in vivo and 3T3-L1 adipocytes in vitro, it appears likely that the observed AMPK activation in human adipose occurs, at least in part, in the adipocyte fraction.

As AMPK activation in adipocytes has been associated with attenuated lipolysis [6-8, 35], attenuated differentiation of pre-adipocytes into mature adipocytes $[13,46]$ and reduced insulin-stimulated glucose transport in adipocytes [14-16], such effects may act to divert energy away from storage as lipid and stimulate glucose use by skeletal muscle. Furthermore, as stimulation of AMPK in vascular cells and macrophages has been demonstrated to elicit antiinflammatory effects that would also be beneficial in type 2 diabetes [28, 45, 47, 48], stimulation of adipose AMPK in both the adipocyte and stromal/vascular cell fractions of adipose may explain some of the beneficial metabolic effects of metformin in patients with type 2 diabetes.

Acknowledgements This work was supported by a British Heart Foundation project grant (PG/03/114/16038), TENOVUS-Scotland, a Biotechnology and Biological Sciences Research Council PhD Studentship (P. J. Logan) and a Diabetes UK R. D. Lawrence Fellowship (I. P. Salt).

Duality of interest The authors declare that there is no duality of interest associated with this manuscript.

\section{References}

1. Zhou G, Myers R, Li Y et al (2001) Role of AMP-activated protein kinase in mechanism of metformin action. J Clin Invest 108:11671174

2. Shaw RJ, Lamia KA, Vasquez D et al (2005) The kinase LKB1 mediates glucose homeostasis in liver and therapeutic effects of metformin. Science 310:1642-1646

3. UK Prospective Diabetes Study (UKPDS) Group (1998) Effect of intensive blood-glucose control with metformin on complications in overweight patients with type 2 diabetes (UKPDS 34). Lancet 352:854-865

4. Hardie DG (2008) AMPK: a key regulator of energy balance in the single cell and the whole organism. Int J Obes 32:S7-S12

5. Zhang BB, Zhou G, Li C (2009) AMPK: an emerging drug target for diabetes and the metabolic syndrome. Cell Metab 9:407-416

6. Sullivan JE, Brocklehurst KJ, Marley AE, Carey F, Carling D, Beri RK (1994) Inhibition of lipolysis and lipogenesis in isolated rat adipocytes with AICAR, a cell-permeable activator of AMPactivated protein kinase. FEBS Lett 353:33-36

7. Garton AJ, Yeaman SJ (1990) Identification and role of the basal phosphorylation site on hormone-sensitive lipase. Eur J Biochem 191:245-250

8. Corton JM, Gillespie JG, Hawley SA, Hardie DG (1995) 5aminoimidazole-4-carboxamide ribonucleoside. A specific method for activating AMP-activated protein kinase in intact cells? Eur J Biochem 229:558-565

9. Daval M, Diot-Dupuy F, Bazin R et al (2005) Anti-lipolytic action of AMP-activated protein kinase in rodent adipocytes. J Biol Chem 280:25250-25257
10. Koh HJ, Hirshman MF, He $\mathrm{H}$ et al (2007) Adrenaline is a critical mediator of acute exercise-induced AMP-activated protein kinase activation in adipocytes. Biochem J 403:473-481

11. Gauthier MS, Miyoshi H, Souza SC et al (2008) AMP-activated protein kinase is activated as a consequence of lipolysis in the adipocyte: potential mechanism and physiological relevance. J Biol Chem 283:16514-16524

12. Villena JA, Viollet B, Andreelli F, Kahn A, Vaulont S, Sul HS (2004) Induced adiposity and adipocyte hypertrophy in mice lacking the AMP-activated protein kinase-alpha2 subunit. Diabetes 53:2242-2249

13. Habinowski SA, Witters LA (2001) The effects of AICAR on adipocyte differentiation of 3T3-L1 cells. Biochem Biophys Res Commun 286:852-856

14. Salt IP, Connell JM, Gould GW (2000) 5-Aminoimidazole-4carboxamide ribonucleoside (AICAR) inhibits insulin-stimulated glucose transport in 3T3-L1 adipocytes. Diabetes 49:1649-1656

15. Gaidhu MP, Fediuc S, Ceddia RB (2006) 5-Aminoimidazole-4carboxamide-1-beta-D-ribofuranoside-induced AMP-activated protein kinase phosphorylation inhibits basal and insulinstimulated glucose uptake, lipid synthesis, and fatty acid oxidation in isolated rat adipocytes. J Biol Chem 281:25956-25964

16. Gaidhu MP, Perry RL, Noor F, Ceddia RB (2010) Disruption of AMPK $\alpha 1$ signaling prevents AICAR-induced inhibition of AS160/TBC1D4 phosphorylation and glucose uptake in primary rat adipocytes. Mol Endocrinol 24:1434-1440

17. Chavez JA, Roach WG, Keller SR, Lane WS, Lienhard GE (2008) Inhibition of GLUT4 translocation by Tbc1d1, a Rab GTPaseactivating protein abundant in skeletal muscle, is partially relieved by AMP-activated protein kinase activation. J Biol Chem 283:9187-9195

18. Park H, Kaushik VK, Constant S et al (2002) Coordinate regulation of malonyl-CoA decarboxylase, sn-glycerol-3-phosphate acyltransferase, and acetyl-CoA carboxylase by AMPactivated protein kinase in rat tissues in response to exercise. J Biol Chem 277:32571-32577

19. Sponarova J, Mustard KJ, Horakova O et al (2005) Involvement of AMP-activated protein kinase in fat depot-specific metabolic changes during starvation. FEBS Lett 579:6105-6110

20. LeBrasseur NK, Kelly M, Tsao TS et al (2006) Thiazolidinediones can rapidly activate AMP-activated protein kinase in mammalian tissues. Am J Physiol Endocrinol Metab 291:E175-E181

21. Kola B, Hubina E, Tucci SA et al (2005) Cannabinoids and ghrelin have both central and peripheral metabolic and cardiac effects via AMP-activated protein kinase. J Biol Chem 280:25196-25201

22. Christ-Crain M, Kola B, Lolli F et al (2008) AMP-activated protein kinase mediates glucocorticoid-induced metabolic changes: a novel mechanism in Cushing's syndrome. FASEB J 22:1672-1683

23. Gaidhu MP, Anthony NM, Patel P, Hawke TJ, Ceddia RB (2010) Dysregulation of lipolysis and lipid metabolism in visceral and subcutaneous adipocytes by high-fat diet: role of ATGL, HSL, and AMPK. Am J Physiol Cell Physiol 298:C961C971

24. Kola B, Christ-Crain M, Lolli F et al (2008) Changes in adenosine 5 -monophosphate-activated protein kinase as a mechanism of visceral obesity in Cushing's syndrome. J Clin Endocrinol Metab 93:4969-4973

25. Ota S, Horigome K, Ishii T et al (2009) Metformin suppresses glucose-6-phosphatase expression by a complex I inhibition and AMPK activation-independent mechanism. Biochem Biophys Res Commun 388:311-316

26. Foretz M, Hébrard S, Leclerc J et al (2010) Metformin inhibits hepatic gluconeogenesis in mice independently of the LKB1/AMPK pathway via a decrease in hepatic energy state. J Clin Invest 120:2355-2369 
27. Musi N, Hirshman MF, Nygren J et al (2002) Metformin increases AMP-activated protein kinase activity in skeletal muscle of subjects with type 2 diabetes. Diabetes 51:2074-2081

28. Boyle JG, Logan PJ, Ewart MA et al (2008) Rosiglitazone stimulates nitric oxide synthesis in human aortic endothelial cells via AMP-activated protein kinase. J Biol Chem 283:11210-11217

29. Nelson SM, Freeman DJ, Sattar N, Lindsay RS (2008) Role of adiponectin in matching of fetal and placental weight in mothers with type 1 diabetes. Diabetes Care 31:1123-1125

30. Huang YC, Chang WL, Huang SF, Lin CY, Lin HC, Chang TC (2010) Pachymic acid stimulates glucose uptake through enhanced GLUT4 expression and translocation. Eur J Pharmacol 648:39-49

31. Wu X, Motoshima H, Mahadev K, Stalker TJ, Scalia R, Goldstein BJ (2003) Involvement of AMP-activated protein kinase in glucose uptake stimulated by the globular domain of adiponectin in primary rat adipocytes. Diabetes 52:1355-1363

32. Phillips SA, Ciaraldi TP, Kong APS et al (2003) Modulation of circulating and adipose tissue adiponectin levels by antidiabetic therapy. Diabetes 52:667-674

33. Drzewoski J, Zurawska-Klis M (2006) Effect of gliclazide modified release of adiponectin, interleukin- 6 , and tumor necrosis factor-alpha plasma levels in individuals with type 2 diabetes mellitus. Curr Med Res Opin 22:1921-1926

34. Huypens P, Quartier E, Pipeleers D, van de Casteele M (2005) Metformin reduces adiponectin protein expression and release in 3T3-L1 adipocytes involving activation of AMP activated protein kinase. Eur J Pharmacol 518:90-95

35. Bourron O, Daval M, Hainault I et al (2010) Biguanides and thiazolidinediones inhibit stimulated lipolysis in human adipocytes through activation of AMP-activated protein kinase. Diabetologia 53:768-778

36. Hong Y, Rohatagi S, Habtemariam B, Walker JR, Schwartz SL, Mager DE (2008) Population exposure-response modeling of metformin in patients with type 2 diabetes mellitus. J Clin Pharmacol 48:696-707

37. Ranganathan G, Unal R, Pokrovskaya I et al (2006) The lipogenic enzymes DGAT1, FAS, and LPL in adipose tissue: effects of obesity, insulin resistance, and TZD treatment. J Lipid Res 47:2444-2450
38. Ciaraldi TP, Kong APS, Chu NV (2002) Regulation of glucose transport and insulin signaling by troglitazone or metformin in adipose tissue of type 2 diabetic subjects. Diabetes 51:30-36

39. Abbasi F, Carantoni M, Chen YI, Reaven GM (1998) Further evidence for a central role of adipose tissue in the antihyperglycemic effect of metformin. Diabetes Care 21:1301-1305

40. James AP, Watts GF, Mamo JC (2005) The effect of metformin and rosiglitazone on postprandial lipid metabolism in obese insulin-resistant subjects. Diab Obes Metab 7:381-389

41. Mooney MH, Fogarty S, Stevenson C et al (2008) Mechanisms underlying the metabolic actions of galegine that contribute to weight loss in mice. Br J Pharmacol 153:1669-1677

42. Fischer M, Timper K, Radimerski T et al (2010) Metformin induces glucose uptake in human preadipocyte-derived adipocytes from various fat depots. Diab Obes Metab 12:356-359

43. Jensterle M, Janez A, Mlinar B, Marc J, Prezelj J, Pfeifer M (2008) Impact of metformin and rosiglitazone treatment on glucose transporter $4 \mathrm{mRNA}$ expression in women with polycystic ovary syndrome. Eur J Endocrinol 158:793-801

44. Kukidome D, Nishikawa T, Sonoda K et al (2006) Activation of AMP-activated protein kinase reduces hyperglycemia-induced mitochondrial reactive oxygen species production and promotes mitochondrial biogenesis in human umbilical vein endothelial cells. Diabetes 55:120-127

45. Nath N, Khan M, Paintlia MK, Singh I, Hoda MN, Giri S (2009) Metformin attenuated the autoimmune disease of the central nervous system in animal models of multiple sclerosis. J Immunol 182:8005-8014

46. Dagon Y, Avraham Y, Berry EM (2006) AMPK activation regulates apoptosis, adipogenesis, and lipolysis by eIF $2 \alpha$ in adipocytes. Biochem Biophys Res Commun 340:43-47

47. Ewart MA, Kohlhaas CF, Salt IP (2008) Inhibition of TNF $\alpha$ stimulated monocyte adhesion to human aortic endothelial cells by AMP-activated protein kinase. Arterioscler Thromb Vasc Biol 28:2255-2257

48. Yang Z, Kahn BB, Shi H, Xue BZ (2010) Macrophage $\alpha 1$ AMPactivated protein kinase $(\alpha 1 \mathrm{AMPK})$ antagonizes fatty acidinduced inflammation through SIRT1. J Biol Chem 285:1905119059 